\title{
Diseño de una plataforma virtual 3D para la formación de personas con discapacidad
}

\section{Design of a $3 \mathrm{D}$ virtual platform for the training of people with disabilities}

Recibido: agosto 20 de 2018 | Revisado: setiembre 15 de 2018 | Aceptado: octubre 02 de 2018

\author{
Augusto Bernuy-Alba ${ }^{\mathrm{I}}$ \\ Amparo Cervantes Arriola ${ }^{\mathrm{I}}$
}

\begin{abstract}
RESUMEN
El estudio analiza las necesidades de las personas con discapacidad física para tener igualdad de oportunidades en una formación técnica considerando en cuenta su nivel de multiculturalidad y protocolos de comunicación relacionados con su condición de discapacidad. La finalidad del estudio es avanzar hacia la inserción productiva en el mercado nacional e internacional de la Personas con Discapacidad (PCD) locomotora a través de la creación de un modelo de aprendizaje virtual basado en diseño tecnológico de agentes inteligentes de software en mundos virtuales. La investigación considera 3 etapas conceptuales: primero, el análisis del estado del arte conducente a crear, diseñar y desarrollar el modelo tecnológico, segundo la inducción cultural y técnica de las PCD en la nueva plataforma tecnológica y su formación en temáticas definidas en un proyecto piloto y tercero el diseńo de una plataforma tecnológica inteligente para el aprendizaje virtual en un escenario controlado basado en agentes inteligentes de software que permitirán generar conocimientos útiles para la persona que se beneficia. Los resultados permitirán construir aplicaciones de aprendizaje virtual dirigido con diálogos inteligentes para la formación productiva de personas con discapacidad física.
\end{abstract}

Palabras clave: capacitación productiva, aprendizaje virtual, formación de personas con discapacidad

\section{Abstract}

The study analyzes needs of people with physical disabilities to get equal opportunities in a minor learning based on the level of multiculturalism and communication protocols related to their disability status. The goal is move towards the insertion into both, the local and international market, of People with Disabilities (PWD) through the creation of a virtual learning model based on technological design of intelligent software agents in virtual worlds. The research has three stages: first, the analysis of the state of the art leading to create, design and develop the technological model, second the cultural and technical induction of the PWD in the new technological platform and its formation in topics defined in a pilot project and third, the last one is design of technological platform for virtual learning based on intelligent software agents that will generate useful knowledge for the person who will be benefited. The result will allow to build applications of virtual learning running with intelligent dialogues for the productive formation of people with physical disability

Key words: learning for work, e-learning, learning for people with disabilities
Universidad de San Martín de Porres. Lima - Perú

Facultad de Ingeniería y Arquitectura abernuya@usmp.pe acervantesarriola@gmail.com

https://doi.org/10.24265/campus.2018.v23n26.06

| Campus | Lima, perú | V. XXIII | N. 26 | PP. I6I-I74 | EJULIO-DiCIEMbRE | 20I8 | ISSN I8I2-6049 


\section{Introducción}

Las personas con discapacidad tienen que enfrentar numerosas barreras para poder insertarse de manera competitiva en el mercado: prejuicio social, problemas de accesibilidad, un sistema educativo que no las contempla y empresas que no están preparadas para recibirlas. Una ciudad que no los contempla desde su arquitectura y transporte público, un sistema educativo que los expulsa, un mercado laboral que no se ajusta a sus posibilidades, un Estado que no genera las políticas públicas necesarias para acompańarlos y una sociedad que les da la espalda. En consecuencia, las personas con discapacidad están conjeturadas de no ser buenos profesionales. Y en la mayoría de los casos esta afirmación es cierta. No porque no puedan llegar a serlo, sino porque las condiciones sociales lamentablemente no lo permite. Sin embargo, y contra todos los pronósticos, algunos se abren camino en el mercado laboral y prueban que, con los apoyos necesarios, es posible.

Las personas con discapacidad representan aproximadamente mil millones de personas, es decir un $15 \%$ de la población mundial. Alrededor del 80 por ciento están en edad de trabajar. Sin embargo, su derecho a un trabajo decente, es con frecuencia denegado. Las personas con discapacidad, en particular las mujeres se enfrentan a enormes barreras actitudinales, físicas y de información que dificultan el disfrute a la igualdad de oportunidades en el mundo laboral. (ILO, 2014): Un dato significativo es que el $80 \%$ de las personas con discapacidades son desempleadas. (Rojas, 2003). La Organización Mundial de la Salud a través de su Informe Mundial Sobre la Discapacidad (OMS, 2011) opina que "la discapacidad no es un atributo de la persona, sino un complicado conjunto de condiciones, muchas de las cuales son creadas por el ambiente social. Por lo tanto, el manejo del problema requiere la actuación social y es responsabilidad colectiva de la sociedad hacer las modificaciones ambientales necesarias para la participación plena de las personas con discapacidad en todas las áreas de la vida social. La cuestión se sitúa, por lo tanto, en el nivel de las actitudes y de la ideología, y requiere cambios sociales, los cuales se transforman en el nivel político en una cuestión de derechos humanos. Según este modelo, la discapacidad se configura como un tema de índole político".

La primera encuesta nacional especializada sobre discapacidad realizada en el año 2012 por el Instituto Nacional de Estadística e Informática (INEI, 2012) a nivel nacional, en el área urbana y rural fue aplicada en 22,657 viviendas particulares a nivel nacional. El 5,2\% de la población nacional ( 1 millón $575 \mathrm{mil}$ 402 personas) padecen de algún tipo de discapacidad o limitación física y/o mental. Esta condición afecta, en mayor proporción, a la población $\geq 65$ años $(50,4 \%)$ y entre 15 a 64 años $(41,3 \%)$. Los tipos de limitación más frecuentes en la población con discapacidad es la dificultad motora es decir, moverse o caminar y/o para usar brazos o piernas $(59,2 \%)$ y las de tipo visual $(50,9 \%)$, y en la mayoría de los caso existen limitaciones múltiples. El 40,4\% de personas con discapacidad tienen nivel educativo primario, el 22,4\% estudios secundarios, $11,6 \%$ estudios superiores univer- 
sitarios y no universitarios y el $22,3 \%$ no tiene nivel educativo. La población con alguna discapacidad del área rural ha alcanzado menores niveles de estudios respecto a los que residen en el área urbana. (INEI, 2012).

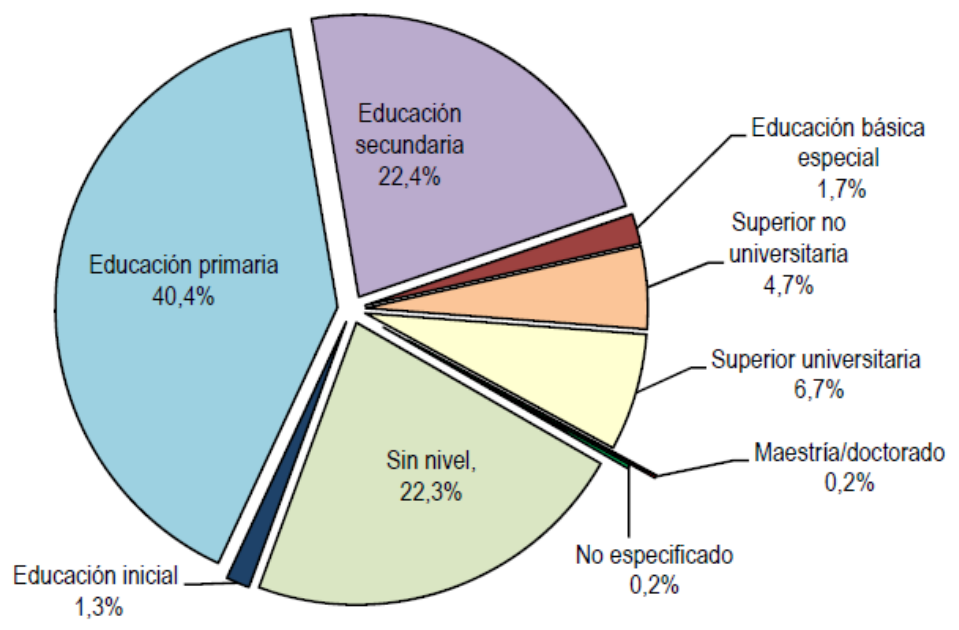

Figura 1. Personas con discapacidad $\geq 3$ años de edad, según nivel educativo. Fuente: INEI - Primera Encuesta Nacional Especializada sobre Discapacidad 2012.

El 22\% de personas $\geq 14$ años de edad con alguna discapacidad, se encuentra trabajando o buscando trabajo, pertenecen a la Población Económicamente Ac- tiva. En tanto, que el 76,8\% corresponde a la Población Económicamente Inactiva y el $1,5 \%$ no especificó su condición de ocupación.

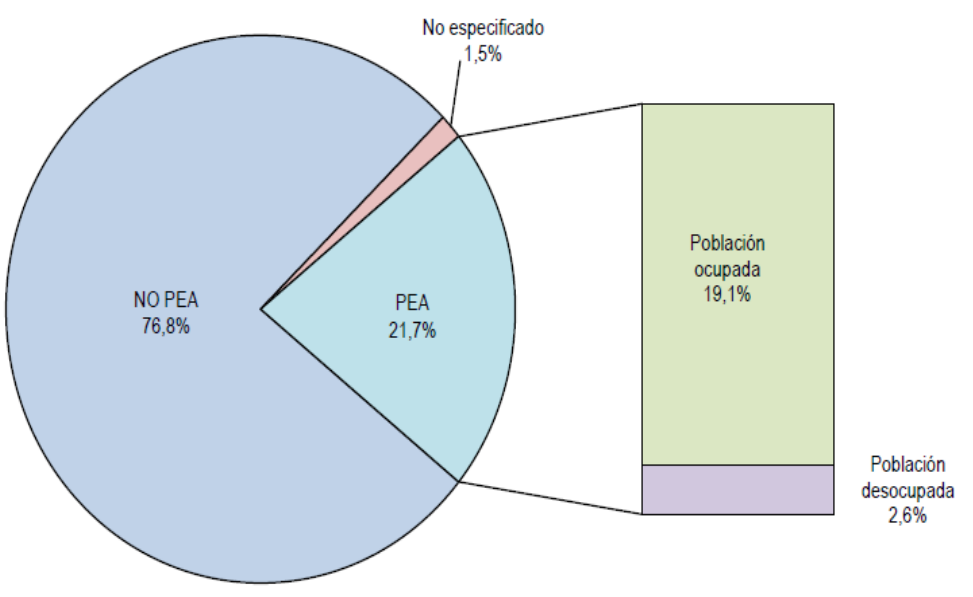

Figura 2. Actividad económica, Personas con discapacidad $\geq 14$ años de edad. Fuente: INEI - Primera Encuesta Nacional Especializada sobre Discapacidad 2012.

Del total de la Población Económicamente Activa (PEA) $\geq 14$ años de edad con discapacidad, el $87,9 \%$ se encuentra ocupada y se desempeñan en el área labo- ral como: trabajadores no calificados de los servicios $(28,4 \%)$, trabajadores calificados de los servicios personales $(18,2 \%)$, agricultores y trabajadores agropecuarios 
calificados $(14,4 \%)$, obreros, operadores de las actividades mineras, canteras $(8,8 \%)$, obrero de construcción, confeccionadores de productos $(6,7 \%)$ y como profesionales, científicos e intelectuales (5,3\%), entre las principales ocupaciones. El 12,1\% de la PEA con discapacidad se encuentra desocupado.

Las PCD no solo reciben malos tratos por parte de la sociedad, más allá de ello tienen que afrontar las necesidades que no se les brinda con facilidad, como la educación y un empleo factible acorde a su situación, las limitaciones que tienden a presentar los estudiantes con discapacidades físicas durante el periodo de aprendizaje educacional suelen ser, el rechazo, timidez, y desconcentración, muchas veces son causadas por la falta de comunicación, por mantenerse alejados de los malos tratos y en algunas ocasiones burlas y acosos que afrontan. Por otro lado, son muy pocas las PCD que logran superar el nivel de Educación Secundario y las oportunidades que tienen para poder llevar estudios superiores están lamentablemente fuera del alcance.

La educación no solo es un valor, sino también, un derecho y, por lo tanto, es exigible a los Estados. Está consagrado a nivel constitucional y en distintos instrumentos internacionales por lo que se erige como una obligación al Estado a todo nivel. La educación como derecho económico, social y cultural es también un derecho para las personas con discapacidad. No se limita solo a la educación primaria y secundaria, sino que se extiende a la educación superior. Ahora bien, es innegable que las personas con discapacidad han sufrido una estigmatización social que se ha expresado claramente en el impedimento social de acceso a una educación superior. Esta problemática ha sido específicamente abordada por la Convención sobre los Derechos de las Personas con Discapacidad (CDPCD) que analizaremos a lo largo del presente trabajo. (Lazarte \& Renato Constantino Caycho, 2013)

El problema planteado es:

¿Cómo se puede ayudar a solucionar el problema de formación productiva a las personas con discapacidad física con una educación superior, en niveles técnicos y en igualdad de oportunidades?

\section{Objetivos}

\section{Objetivo General}

El diseño de un centro de excelencia virtual en 3D para la formación productiva de personas con discapacidad física a fin de promover su formación y empleo en igualdad de oportunidades basado en diseño de agentes inteligentes y la gestión del conocimiento.

\section{Objetivos específicos}

a) Investigación del perfil de las PCD basado en el estado del arte.

b) Analizar de las características de interculturalidad y requerimientos para sus procesos de formación en oficios y de forma independiente.

c) Diseñar los componentes de un modelo y una arquitectura tecnológica para la formación productiva en mundos virtuales.

\section{Estado del Arte}

Romero, Sperduti, \& others. en el 2005 realizaron un estudio cuyo obje- 
tivo fue orientado a estudiar el E-learning como herramienta para la capacitación del personal, su importancia se centró en que permite que la persona se haga responsable de su capacitación, que los conocimientos adquiridos sean actualizados, mejorando así la calidad del aprendizaje y el uso de tecnología multimedia e Internet. El estudio realizado es de tipo documental a un nivel descriptivo. Se utilizó información bibliográfica de una manera precisa y que define los aspectos y características más importantes sobre el E-learning. Las teorías del constructivismo, de la conversación y del conocimiento situado son enfoques que plantean elementos importantes para el aprendizaje los cuales están presentes en el E-learning.

Rodríguez, Alvarado y Moreno en el 2007, presenta un modelo socio ecológico de inclusión social dirigido a personas en situación de discapacidad, sus cuidadores y la comunidad de la vereda $\mathrm{La}$ Balsa del Municipio de Chía, el cual fue construido a través de una experiencia de investigación acción participativa. En la primera fase del proyecto, se identificaron los significados que han construido las personas, sus familias y las redes de apoyo alrededor de la discapacidad, los cuales están relacionados con los problemas que deben afrontar en su vida cotidiana y revelan que esta condición les permite reconocer su valía y potencialidades.

En la segunda fase participaron 42 personas en situación de discapacidad, sus cuidadores, líderes comunitarios y un equipo conformado por profesionales de enfermería, psicología y pedagogía, la cual estuvo dirigida a construir partici- pativamente el modelo a través de foros comunitarios. Además del uso de estrategias como las metáforas del árbol y del sol que tienen un carácter evaluativo y motivacional, a partir de las cuales se identificaron los núcleos de desarrollo del modelo: personal, familiar, comunitario, de comunicación y de bienestar, los cuales se utilizan en la tercera fase del proyecto.

Almada en el 2000, por un lado, aborda el tema de los nuevos requisitos en materia de formación profesional de la mano de obra, que surgen como resultado de la innovación tecnológica y de las nuevas formas de organización del trabajo y, por otra parte, se hace una presentación del funcionamiento, las actividades desarrolladas y los logros alcanzados por el Consejo de Normalización y Certificación de Competencia Laboral (CONOCER) de México.

\section{Desarrollo de aplicaciones educativas}

Un estudio realizado por el Departamento de Informática de la Universidad Carlos III de Madrid, describe una sociedad donde la tecnología ha avanzado a tal punto que brinda la posibilidad de acceder a contenidos educativos en la red. No obstante, en el desarrollo de dichas tecnologías, es poco común que se considere dentro de su diseño la posibilidad de utilización por parte de personas con discapacidad. Es por ello, que surge la iniciativa de emplear Sistemas Multiagentes, Sistemas de Diálogos y Mundos Virtuales para desarrollar una plataforma educativa óptima para el uso de personas con discapacidad. Dichos sistemas virtuales deben ser intuitivos e inteligentes, y además, se debe asegurar una comunicación eficaz entre el usuario y el sistema, 
lo cual se puede lograr a través de Tecnologías de procesamiento de lenguaje, para así incorporar capacidades comunicativas inteligentes en los sistemas. Según el estudio, es fundamental que estos sistemas de aprendizaje se expandan hacia las personas con discapacidades motoras, apoyándose en los mundos virtuales y agentes inteligentes, los cuales deben ser capaces de comunicarse con el usuario, otros agentes y programas.

Se propone el uso de tecnologías de mundos virtuales desarrollados mediante Open Simulator, como Sloodle, para los ambientes virtuales como aulas y edificios, integrando Moodle como gestor de aprendizaje (Griol et al., 2014).

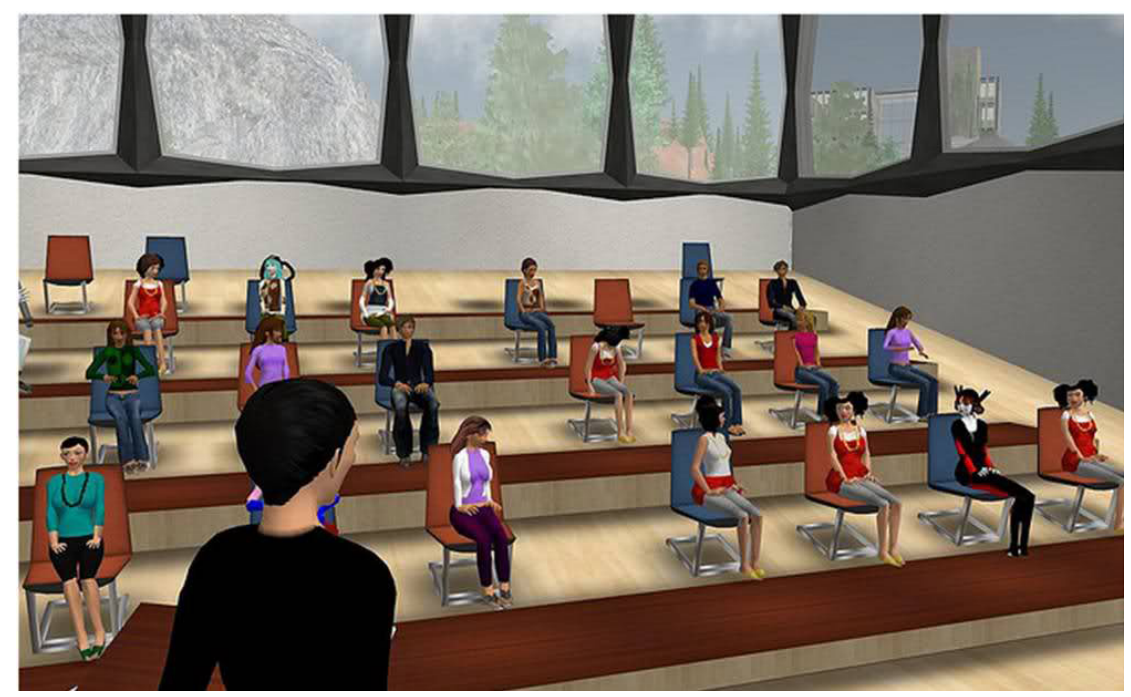

Figura 3. Interfaz de Sloodle. Elaboración: (Hernández, 2011)

\section{La educación y el empleo a distancia}

De acuerdo al estudio realizado por Barés y Litovicios, en el 2009, la educación telemática, (sistemas de información y telecomunicaciones aplicado en la educación), ha evolucionado desde la década de los 80, expandiéndose debido al incremento del uso de computadoras personales y la Enseñanza Asistida por Ordenador (EAO). Es este entorno el que está mejor calificado para ser usado por las PCD, debido a que es de fácil acceso e inclusivo. Sin embargo, un punto a considerar es que al margen de la evolución tecnológica, debe existir un avance en el modo de trabajo de los docentes en relación a las PCD para un mejor uso de la informática, ya que de lo contrario di- chas personas no tendrán la oportunidad de obtener nuevas fuentes de desarrollo personal.

Nuestro estudio plantea desarrollar propuestas innovadoras orientadas a mejorar las competencias de las PCD, por medio de dos casos: el primero el de "Diseño Gráfico y Creatividad hacia Personas con Discapacidad", y segundo el de "Implementación de una Plataforma de Educación a Distancia Inclusiva”. En ambos casos Barés y Litovicios, en el 2009, enfatiza, la importancia de considerar las limitaciones que posee una PCD y que deben recibir un trato individual y personalizado. El estudio se apoya en el uso de Sistemas de Gestión de Aprendizaje (LMS) para poder llevar un control de 
cómo se dicta un curso de manera virtual, además de recolectar información del nivel de satisfacción de una persona con el curso, de las dificultades que encontró, si logró adquirir conocimientos, los tiempos estuvieron bien planificados, el nivel de relación con el tutor, entre otros.

\section{Conceptos de capacitación y capacita- ción productiva}

Bohlander y Snell en el 2008 definen a la capacitación como "la generalidad de los esfuerzos iniciados por una organización para impulsar el Detección de Necesidades de Capacitación y aprendizaje de sus miembros", mencionan también que la capacitación se refiere a cuestiones de desempeño de corto plazo y desarrollo a largo plazo.

Para Gutiérrez n el 2004, la capacitación es una:

actividad sistemática, planificada $y$ permanente, cuyo propósito general es preparar, desarrollar e integrar a los recursos humanos al proceso productivo, mediante la entrega de conocimientos, desarrollo de habilidades y actitudes necesarias para el mejor desempeño de todos los trabajos en sus actuales y futuros cargos y adaptarlos a las exigencias cambiantes del entorno.

Según Romero B. y Sperduti, S. en el 2005, la capacitación "es la acción destinada a incrementar las actitudes y los conocimientos del trabajador con el propósito de prepararlo para desempeñar eficientemente una unidad de trabajo específico e impersonal."

Por lo expuesto, la capacitación productiva es una actividad sistemática, planificada cuyo propósito es preparar, desarrollar e integrar a los recursos humanos al proceso productivo, mediante la entrega de conocimientos, desarrollo de habilidades y actitudes necesarias el desempeño eficiente en una unidad de trabajo específico e impersonal. La capacitación es una pieza clave para desarrollo de los individuos y las organizaciones, tanto así que incluso en tiempos de crisis esta es indispensable. La diferencia entre capacitación y capacitación productiva radica precisamente en la productividad de la capacitación, es decir en establecer un proceso de definición y seguimiento de resultados.

El binomio capacitación-productividad "es sin duda la clave del desarrollo de nuestro presente y futuro. Existe una capacitación para "hacer", es la más común, la que se puede concebir como el adiestramiento pues persigue "la transformación de la materia”, permite la operación y el manejo de herramientas e instrumentos; pero existe otra capacitación, la más importante, la capacitación para "ser y llegar a ser", esta finca y proyecta el desarrollo del hombre, forma su espíritu de crecimiento, de solidaridad, integra su personalidad dándole su identidad y libertad para optar por valores y caminos de reto y responsabilidad frente sí mismo, su familia, su comunidad y su patria”,Siliceo, A. (2006).

La sociedad actual, enmarcada en la globalización y desarrollo tecnológico, provocan un cambio en el comportamiento y las actitudes frente a situaciones de ajuste, adaptación, transformación y desarrollo, como es el caso de la inclusión de los grupos más vulnerables, en todos los ámbitos de la sociedad, lo 
cual genera la obligación de crear mecanismos que nos permitan sostener el conocimiento y la producción. Siliceo, A. (2006). Una característica de la capacitación productiva es que estos procesos deben estar continuados de proyectos consecuentes de la capacitación, es decir, aplicación inmediata y el seguimiento de habilidades o actitudes de desempeño con el fin de constituir verdaderos programas de cambio y desarrollo. Este seguimiento propiciará la planificación y ejecución de capacitaciones complementarias.

\section{Objetivos de la capacitación pro- ductiva}

La capacitación y la capacitación productiva tienen diversos objetivos dependiendo del enfoque con el que se la desarrolle, es decir, dentro o fuera de una organización. Tomando como base los objetivos que determina Gutiérrez (2014) que se relacionan aquí con el estudio son:

1. Productividad: Aplicable a individuos que pertenecen a una organización dentro de la cual, contribuirán a los objetivos económicos de dicho ente, o a individuos independientes que contribuyen a la consecución de sus propios objetivos económicos habiendo adquirido las competencias para desarrollar tareas particulares.

2. Calidad: Si el individuo ya se encuentra realizando alguna labor dependiente o independiente, un programa de capacitación bien diseñado e implementado contribuirá a elevar la calidad de su producción sea esta bienes tangibles e intangibles o servicios.
3. Beneficio directo: Así como las oportunidades educativas dentro de un centro de trabajo pueden ser consideradas parte del paquete de beneficios, un colaborador independiente calificado por capacitación productiva para realizar un determinado servicios obtendrá más oportunidades de ser requerido en su oficio o profesión y dependiendo de la capacitación en sí misma, oportunidades de desarrollo en otras áreas.

4. Salud y Seguridad: La salud mental y seguridad física de un individuo puede estar relacionada al desarrollo de esfuerzos educacionales como parte de una organización o de forma independiente. Una capacitación adecuada en un ambiente seguro conllevaría a actividades más estables.

5. Prevención de Obsolescencia: Entiéndase a la obsolescencia como la diferencia entre la destreza para realizar un trabajo y la exigencia del mismo. Una capacitación continua mantendrá a los individuos actualizados en el conocimiento y por ende preparados para atender necesidades o requerimientos del mercado laboral.

6. Desarrollo personal: En el ámbito personal los beneficiarios de capacitaciones dentro de una organización o independientes se benefician con conocimientos, competencias y habilidades que se constituyen como indicadores de desarrollo personal.

7. Cambio de Actitud: Implica un cambio de actitud y una mejor y mayor receptividad con respecto de los gru- 
pos de trabajo, además de aumentar la motivación personal.

\section{Diseńo Tecnológico}

\section{Agentes}

Según Russell \& Norvig, (1995), se considera a una entidad como agente inteligente cuando ésta "es capaz de percibir a través de sensores la informaciones que proviene del ambiente donde está insertado y reaccionar a través de efectores", es decir, muestra un comportamiento dependiendo a lo que percibe en el entorno donde se desenvuelve. Las acciones que realizan estos agentes están orientadas a cumplir con los objetivos con los que se ha diseñado.
De acuerdo a Cataldi, Calvo, Salgueiro, \& Lage, (2007) se puede considerar inteligente a un agente cuando este posee características de autonomía y flexibilidad, específicamente en tres principales puntos:

a) Reactividad: Capacidad para percibir su entorno y responder a tiempo a los cambios que ocurren en él.

b) Proactividad: Capacidad de mostrar un comportamiento dirigido por objetivos.

c) Habilidad Social: Capacidad de interactuar con otros agentes inteligentes (software) o personas.

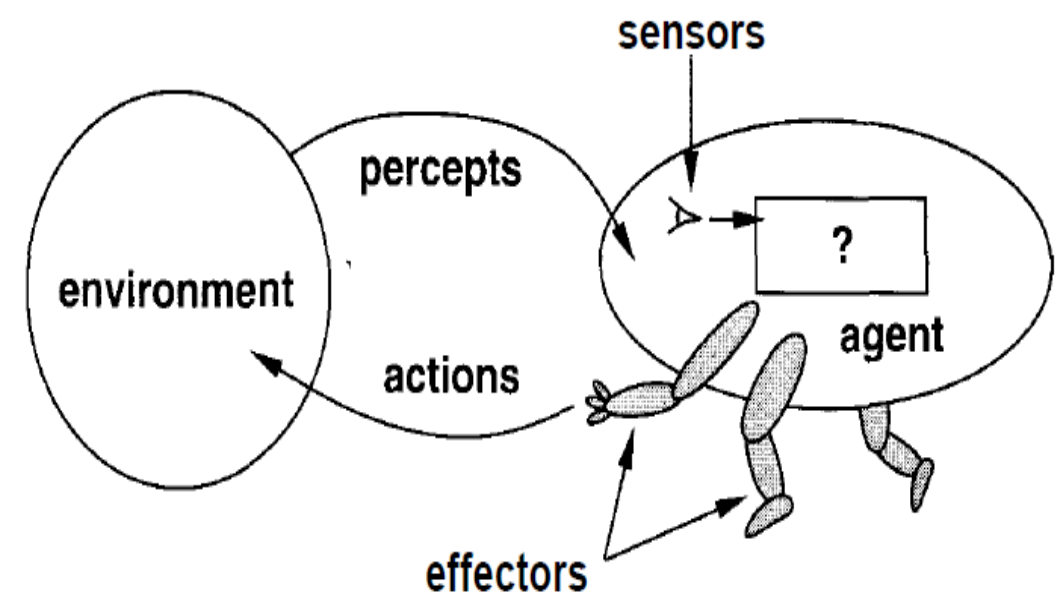

Figura 4. Modelo de interacción de agentes. Elaboración: (Russell \& Norvig, 1995)

La ilustración muestra el modelo de interacción en el que se desenvuelve un agente inteligente. Debe mostrar un comportamiento racional por lo que para cada posible secuencia de percepciones, una agente debería realizar una acción que se espera para maximizar el de su medida. Bernuy \& García, (2006) muestran un procedimiento de en una situación normal en el sistema integrado con el módulo de docente, Figura 5. 


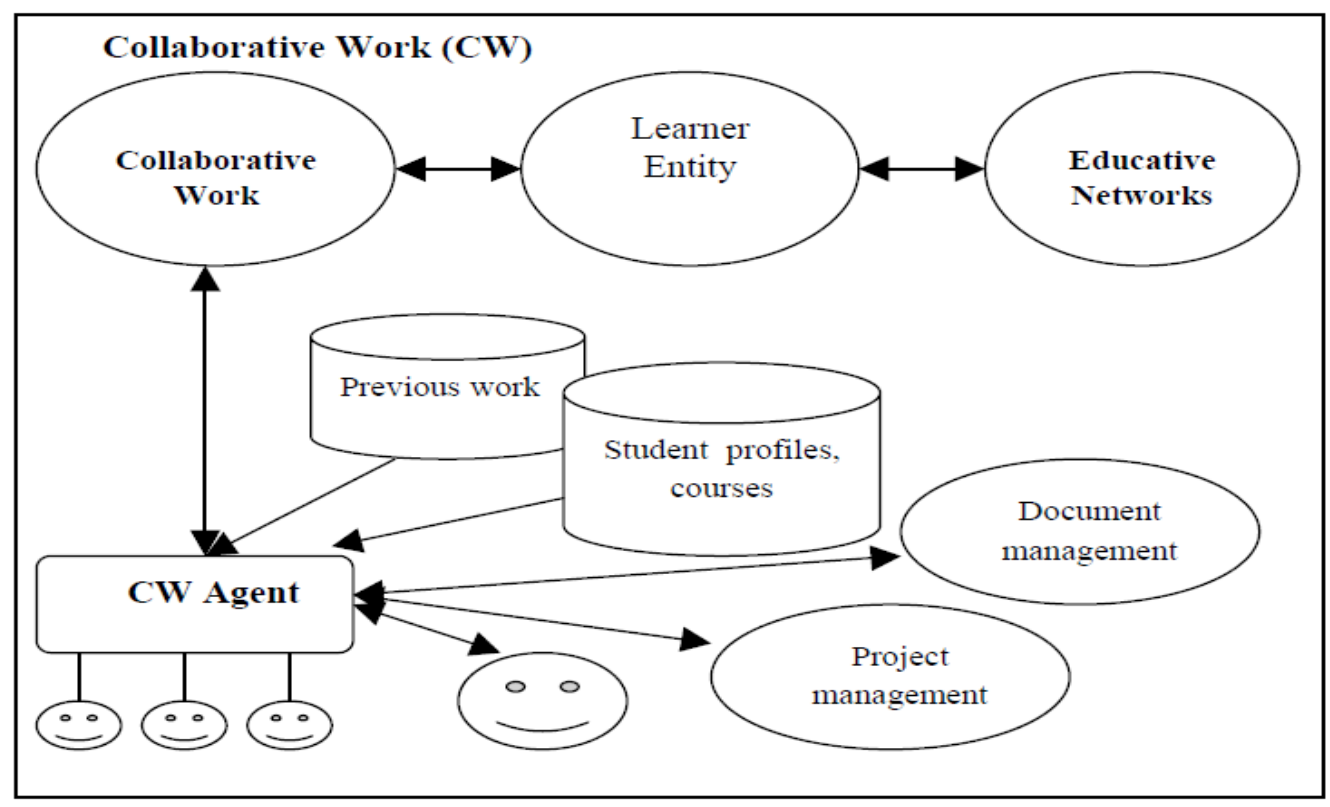

Figura 5. Propuesta de Agentes para Trabajo Colaborativo Elaboración. Bernuy \& García, (2006) "collection-title":"IFIP International Federation for Information Processing","collection-number":"210","publisher":"Springer US","page":"267-271","source": "link.springer.com", "abstract": "The paper presents the basic requirements that must cover distance education processes ("e-learning"

\section{Contextio de modelo}

Se definen las siguientes dimensiones estructurales en la Tabla 1

\section{Tabla 1}

\section{Contenidos de las dimensiones contextuales}

\begin{tabular}{|l|l|l|}
\hline a) & $\begin{array}{l}\text { Dimensión de } \\
\text { diagnóstico y } \\
\text { análisis (Sistema) }\end{array}$ & $\begin{array}{l}\text { Evaluación y detección de necesidades de capacitación. } \\
\text { Evaluación de necesidades de soporte físico (local e implementos) } \\
\text { Perfil del participante } \\
\text { Objetivos de la capacitación }\end{array}$ \\
\hline b) & $\begin{array}{l}\text { Dimensión de } \\
\text { metodología }\end{array}$ & $\begin{array}{l}\text { Programación flexible } \\
\text { Metodología por objetivos y competencias } \\
\text { Didácticas de aprendizaje }\end{array}$ \\
\hline c) & $\begin{array}{l}\text { Desarrollo de contenidos } \\
\text { Contenidos } \\
\text { Diseño de materiales } \\
\text { transformación } \\
\text { Selección e implementación de recursos virtuales (Innovadores, potentes y dinámicos) } \\
\text { Implementación (ejecución) } \\
\text { Orientación tecnológica básica para las PCD } \\
\text { Ejecución y monitoreo } \\
\text { Trabajo colaborativo } \\
\text { Actividades síncronas }\end{array}$ \\
\hline e) & $\begin{array}{l}\text { Conocimientos } \\
\text { resultados }\end{array}$ & $\begin{array}{l}\text { Aptitudes } \\
\text { Capacidades } \\
\text { Evidencias }\end{array}$ \\
\hline
\end{tabular}


De la Tabla 1, se destacan en el modelo, la relación entre las dimensiones de Diseño del Programa y Evaluación, que es la retención y transferencia del conoci- miento en este caso capacidades productivas, así como la relevancia de la tutoría directa (presencial o virtual) en la ejecución.

\section{Módulos de Plataforma 3D}

En este caso el diseño propuesto incluye cuatro (4) módulos, que son descritos en la Tabla 2.

\section{Tabla 2}

Módulos de la plataforma de formación virtual

\begin{tabular}{|c|c|c|c|}
\hline $\mathbf{N}^{\circ}$ & Módulo & Especificación técnica & Entregables \\
\hline 1 & $\begin{array}{l}\text { Sistema de } \\
\text { gestión de } \\
\text { cursos }\end{array}$ & $\begin{array}{l}\text { a) Debe a) Tener un módulo de requisitos y com- } \\
\text { promisos para inscribirse en un curso. } \\
\text { b) Debe tener un módulo de actualización de infor- } \\
\text { mación de la personas con discapacidad } \\
\text { c) Debe tener un módulo para el manejo de las clases } \\
\text { con componentes de diálogos } \\
\text { d) Debe tener un módulo para las evaluaciones con } \\
\text { componentes de diálogos } \\
\text { e) Debe tener un módulo de seguimiento posterior a } \\
\text { la terminación del curso. }\end{array}$ & $\begin{array}{l}\text { Aplicación } \\
\text { Matrícula } \\
\text { Ejecución del curso } \\
\text { Evaluaciones } \\
\text { Seguimiento post curso }\end{array}$ \\
\hline 2 & $\begin{array}{l}\text { Módulo de } \\
\text { profesor }\end{array}$ & $\begin{array}{l}\text { Debe considerar componentes de diálogos. } \\
\text { Debe considerar datos sobre diálogos docente-alumno } \\
\text { en tres contextos: cumplimiento del contenido, } \\
\text { verificación de entendimiento, repaso y consultas }\end{array}$ & $\begin{array}{l}\text { Diálogos de agentes } \\
\text { Datos sobre diálogos } \\
\text { docente alumno en tres } \\
\text { contextos: cumplimiento } \\
\text { del contenido, } \\
\text { verificación de } \\
\text { entendimiento, repaso y } \\
\text { consultas }\end{array}$ \\
\hline 3 & $\begin{array}{l}\text { Módulo de } \\
\text { estudiante }\end{array}$ & $\begin{array}{l}\text { Debe considerar componentes de diálogos. } \\
\text { Debe considerar datos sobre diálogos para búsqueda de } \\
\text { apoyo: con otros estudiantes, con ayudas (comodines) } \\
\text { para hacer ejercicios (explicación paso a paso), con } \\
\text { ayudas para encontrar otros estudiantes en la red. }\end{array}$ & $\begin{array}{l}\text { Diálogos de agentes } \\
\text { Datos sobe diálogos para } \\
\text { búsqueda de apoyo: con } \\
\text { otros estudiantes, con } \\
\text { ayudas (comodines) } \\
\text { para hacer ejercicios } \\
\text { (explicación paso a paso), } \\
\text { con ayudas para encontrar } \\
\text { otros estudiantes en la } \\
\text { red. }\end{array}$ \\
\hline 4 & $\begin{array}{l}\text { Módulo } \\
\text { de objetos } \\
\text { dinámicos }\end{array}$ & $\begin{array}{l}\text { Debe desarrollar objetos móviles 3D para el } \\
\text { usuario Avatares } \\
\text { Debe desarrollar objetos móviles 3D para la gestión } \\
\text { interna: equipos y edificaciones } \\
\text { Debe considerar tipo de objeto, dominio de } \\
\text { pertenencia, características, movilidad, etc. }\end{array}$ & $\begin{array}{l}\text { Movimiento y diálogos } \\
\text { de objetos móviles 3D } \\
\text { para el usuario Avatares } \\
\text { Movimiento y diálogos } \\
\text { de objetos móviles } \\
\text { 3D para la gestión } \\
\text { interna: equipos y } \\
\text { edificaciones }\end{array}$ \\
\hline
\end{tabular}




\section{Resultados de diseño}

El resultado se muestra en las siguientes figuras:

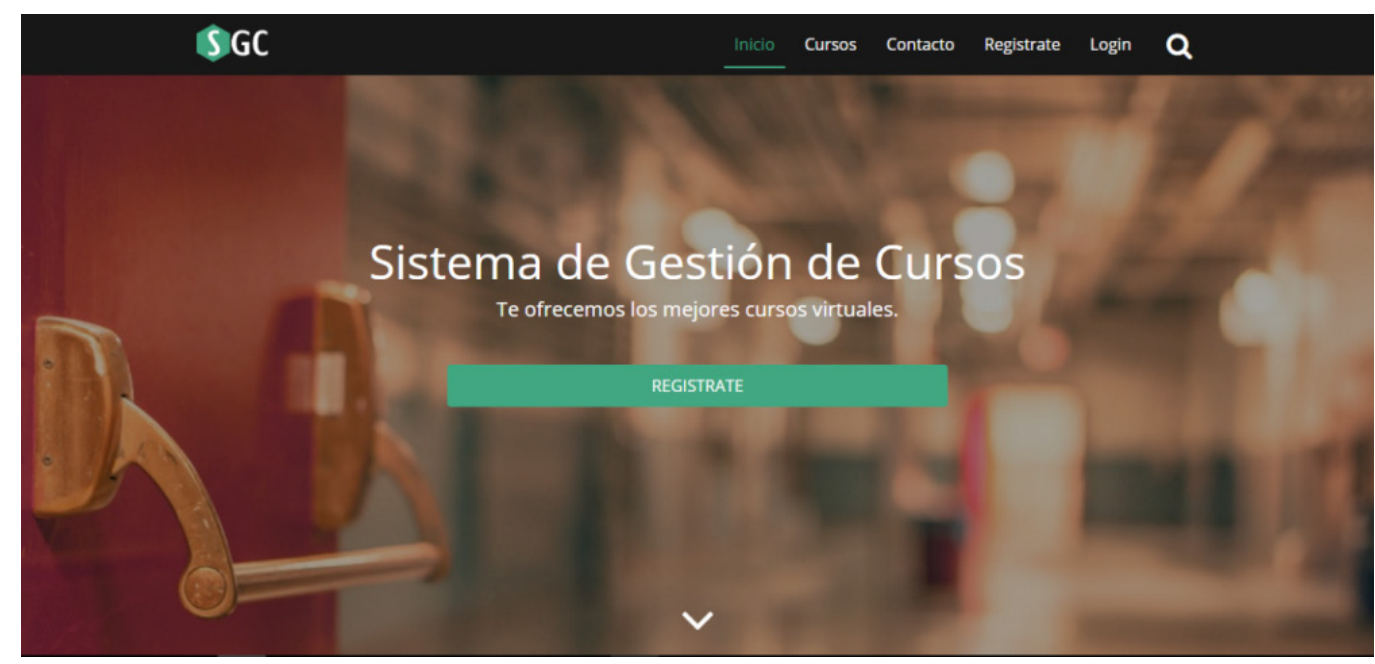

Figura 6. Sistemas de Gestión de Cursos. Elaboración: Propia.

Tabla 3

Diseño de objetos de la Plataforma en 3

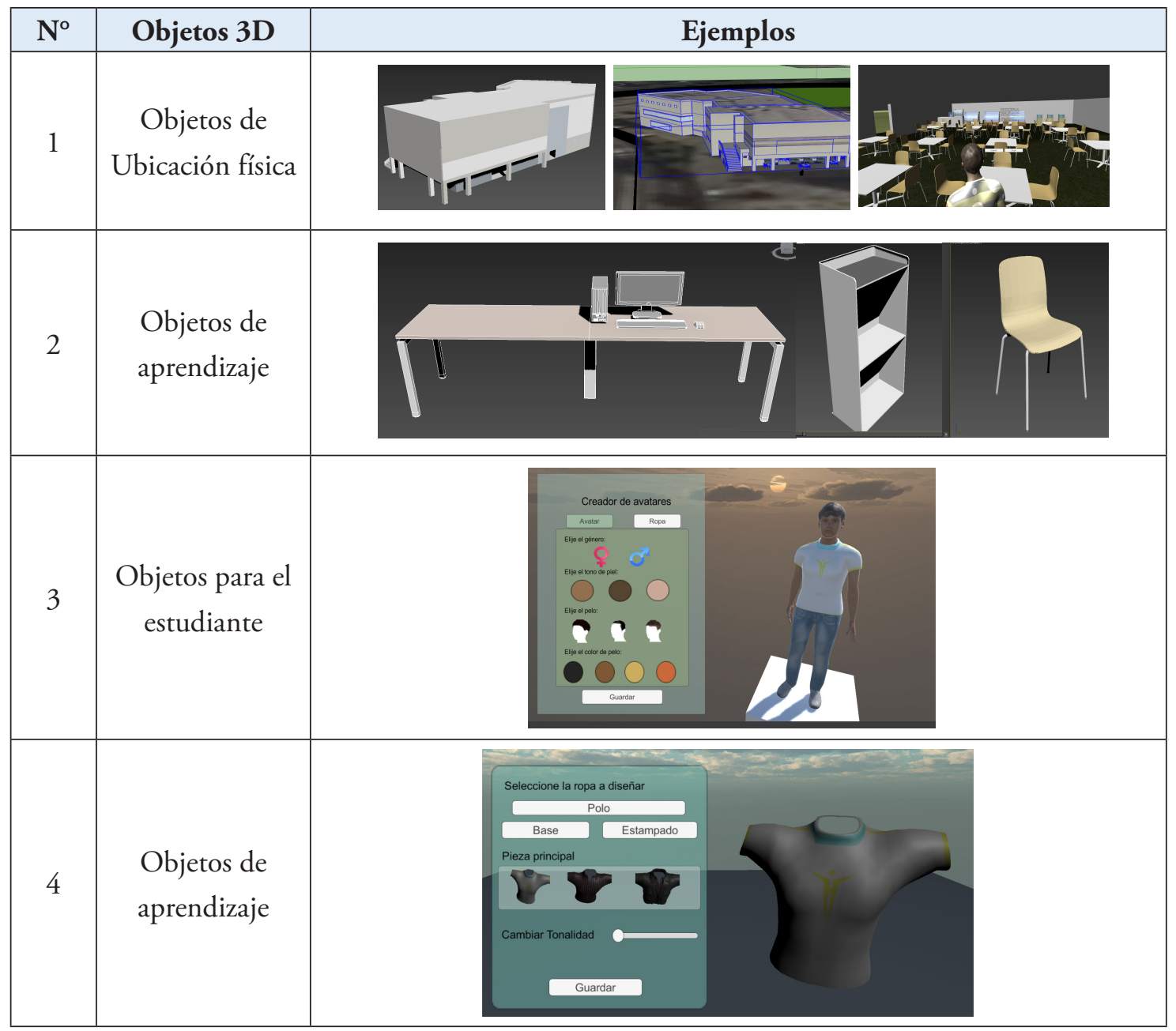




\section{Conclusiones}

Por restricciones de recursos se ha diseńado la plataforma con los siguientes componentes:

i) Aplicación, requisitos de inscripción (duración del curso, tiempo disponible, dedicación y compromiso de terminar,

ii) Análisis de los diálogos principales,

iii) Inicio con clases en PPT, Videos y tareas con las siguientes interfaces:

a) Interface del módulo profesor con el sistema de gestión de cursos;

b) Interface del módulo estudiante con sistema de gestión de cursos; c) Interface del módulo de objetos dinámicos con los módulos de profesor y estudiante.

iv) Estructura para el perfil del usuario con discapacidad: Información sobre el tipo de discapacidad, y sobre el perfil personal en base a una encuesta de partida,

v) Estructura para el compromiso con el curso,

vi) Información sobre la dedicación de tiempo del estudiante para el curso, separada en dedicación semanal y diaria,

vii) Integración con un sistema de recomendaciones.

\section{Referencias}

Almada, A. (2000). Formación de los recursos humanos y competencia laboral. CINTERFOR/OIT. Recuperado a partir de.

http://www.oei.es/etp/formacion_ recursos_humanos_competencia_ laboral_ibarra.pdf

Barés, S., y Litovicus, P., (2009). Capacitación en el uso de las Tecnologías de la Información y las Comunicaciones (TIC), la educación y el empleo a distancia para Personas con Discapacidad. Tecnoeducativa - Tecnologías educativas inclusivas. Ciudad Buenos Aires, Argentina. http://www.telework2014.fidt.org/ wp-content/uploads/2014/09/Capacitaci $\% \mathrm{C} 3 \% \mathrm{~B} 3 n$-en-el-uso-delas-TIC-la-educaci\%C3\%B3n-yel-empleo-a-distancia-para-personas-con-discapa2.pdf
Bernuy, A. y García, V. M. (2006). Collaboration Model in E-Learning for Universities Based on Agents. En D. Kumar \& J. Turner (Eds.), Education for the 21st Century - Impact of ICT and Digital Resources (pp. 267-271). Springer US. Recuperado a partir de http://link.springer.com/chaper/10.1007/978-0-387-347318_29

BOE. (2003). Ley 51/2003, de 2 de diciembre, de igualdad de oportunidades, no discriminación y accesibilidad universal de las personas con discapacidad. núm. 289 miércoles 3 diciembre 2003. http:// www.boe.es/boe/dias/2003/12/03/ pdfs/A43187-43195.pdf 
Bohlander, G., Snell, S., \& Sherman, A. (2008). Administración de recursos humanos. 14 Ed. Cengage Learning Editores. Mexico. http://dip.una. edu.ve/man/administracionrrhh/ paginas/bohlander\%20Cap \%C3\%ADtulo\%20XV.pdf

Cataldi, Z., Calvo, P., Salgueiro, F. A., \& Lage, F. J. (2007). Diseño de Sistemas Tutores Inteligentes con Tecnología de Agentes: Los Agentes Docentes en el Módulo Tutor. Revista Eletrônica de Sistemas de Informação ISSN 1677-3071 doi: 10.5329/RESI, 6(1).

Griol, D., Sanchis, A., Molina, J. M., \& Callejas, Z. (2014). Utilización de las Tecnologías del Habla y de los Mundos Virtuales para el Desarrollo de Aplicaciones Educativas. Procesamiento del Lenguaje Natural, 53(0), 167-170.

Gutiérrez, E., (2004). Diagnóstico de las Necesidades de Capacitación de los Barman de los Hoteles de Cinco Estrellas de Toluca y Metepec. Facultad de Turismo, Universidad Autónoma del Estado de México.

Instituto Nacional de Estadística e Informática. (2012). Primera Encuesta Nacional Especializada sobre Discapacidad. Perú.
Rojas, C. S., \& Rebollo, M. M. R. (2003). Formación a través de las nuevas tecnologías para el fomento de una sociedad más democrática. Edutec. Revista Electrónica de Tecnología Educativa, 0(16). Recuperado a partir de http://www.edutec. es/revista/index.php/edutec-e/article/view/539

Rodríguez, M., Alvarado, A. y Moreno, M. (2007). Construcción participativa de un modelo socioecológico de inclusión social para personas en situación de discapacidad. Acta colombiana de Psicología 10 (2): 181-189, 2007. Universidad de La Sabana, Bogotá - Colombia. http://www.scielo.org.co/pdf/acp/ v10n2/v10n2a17.pdf

Romero, B., Sperduti, S., \& others. (2005). E-learning como herramienta para la capacitación de personal. Recuperado a partir de http://ri.biblioteca.udo.edu.ve/ bitstream/123456789/655/1/TESIS-658.3124_R744_01.pdf

Russell, S. J., \& Norvig, P. (1995). Artificial intelligence: a modern approach. Englewood Cliffs, N.J: Prentice Hall.

Siliceo, A. (2006). Capacitación y desarrollo de personal. 4ta Ed. Editorial Limusa. México

\section{Agradecimientos:}

Al equipo técnico conformado por Miriam Quiroz, Joseph Jiménez y Juan Carlos Contreras 